M.G. Burton, R. Jayawardhana \& T.L. Bourke, eds.

\title{
Adaptive Optics Imaging of Circumstellar Environments
}

\author{
Dániel Apai, Ilaria Pascucci, Hongchi Wang, Wolfgang Brandner and
} Thomas Henning

Max Planck Institute for Astronomy, Königstuhl 17., Heidelberg, Germany D-69117

\section{Carol Grady}

NOAO/STIS, Goddard Space Flight Center, Code 681, NASA/GSFC, Greenbelt, MD 20771, USA

\section{Dan Potter}

Steward Observatory, University of Arizona, 933 N. Cherry Avenue, Tucson, AZ 85721, USA

\begin{abstract}
We present results from our high-resolution, high-contrast imaging campaign targeting the circumstellar environments of young, nearby stars of different masses. The observations have been conducted using the ALFA/CA 3.5m and NACO UT4/VLT adaptive optics systems. In order to enhance the contrast we applied the methods PSF-subtraction and polarimetric differential imaging (PDI). The observations of young stars yielded the identification of numerous new companion candidates, the most interesting one being $\sim 0.5^{\prime \prime}$ from FU Ori. We also obtained high-resolution near-infrared imaging of the circumstellar envelope of SU Aur and AB Aur. Our PDI of the TW Hya circumstellar disk traced back the disk emission as close as $0.1^{\prime \prime} \simeq 6 \mathrm{AU}$ from the star, the closest yet. Our results demonstrate the potential of the adaptive optics systems in achieving high-resolution and high-contrast imaging and thus in the study of circumstellar disks, envelopes and companions.
\end{abstract}

\section{Introduction}

Young, nearby stars are our prime source of information to study the circumstellar disk structure and evolution. They are also the ideal targets for adaptive optics (AO) observations, as they are usually bright enough to provide excellent wavefront reference. The difficulty in directly observing their environment is, however, the large contrast between the central star and any stellar companions, circumstellar envelopes and disks (see also the review by Brandner in this conference).

In the following we present the results from our AO-imaging campaigns targeting nearby, young stars. During these observations we applied different methods to overcome the large contrast between the central star and any companions, envelopes or disks. 


\section{ALFA Observations}

We conducted three observing campaigns (January 2002, October 2002 and June 2003) using the ALFA adaptive optics system mounted at the $3.5 \mathrm{~m}$-telescope at the German-Spanish Observatory in Calar Alto, Spain. The targets were young stars with confirmed infrared excess, mainly focusing on the intermediatemass Herbig Ae/Be stars. The observations aimed to enhance the contrast of the imaging system by the subtraction of a calibrated point spread function (PSF) from the target object. In order to compensate for temporal variations of the PSF due to changes in the atmosphere and in the optical system, we applied short cycles spent alternating on the target and a PSF-comparison star. Each cycle consisted of four dithering positions around the target with roughly 2 minutes total time spent on each position. The basic data reduction has been conducted in the standard fashion with flat field correction and bad pixel removal. Following this, the frames from different dithering positions have been combined to a single image. From each combined image the corrseponding PSFstar was subtracted to remove most of the contribution from the central star. A more detailed description of the data reduction and analyzis can be found in Wang et al. (2003).

\section{ALFA Results}

The PSF-subtraction technique significantly enhanced our capability to detect extended emission and companions. Table 1 summarizes our detections by giving the target names, their coordinates, the type of detections and any further commments. The main results are the detection of numerous new companion candidates and the near-infrared imaging of the nebulosity around SU Aur and AB Aur.

Table 1. Summary of the positive results from the ALFA imaging campaign. The companion candidates of HD 38224 and V380 Cep have been reported before, our observations provide confirmation and the first accurate photometry.

\begin{tabular}{lcccl}
\hline Object & R. A.(J2000) & Dec. (J2000) & Detection & Notes \\
\hline SU Aur & $04^{h} 55^{m} 59.4^{s}$ & $+30^{\circ} 34^{\prime} 01^{\prime \prime} .5$ & Ext. Emission & up to $14^{\prime \prime}$ \\
AB Aur & $04^{h} 55^{m} 45.8^{s}$ & $+30^{\circ} 33^{\prime} 04^{\prime \prime} 3$ & Ext. Emission & \\
V 372 Ori & $05^{h} 34^{m} 47.0^{s}$ & $-05^{\circ} 34^{\prime} 14^{\prime \prime} 6$ & Comp. Candidate & Sep $=9.50^{\prime \prime}$ \\
HD 31305 & $04^{h} 55^{m} 48.2^{s}$ & $+30^{\circ} 20^{\prime} 16^{\prime \prime} 5$ & Comp. Candidate & Sep $=0.42^{\prime \prime}$ \\
HD 282737 & $04^{h} 58^{m} 18.9^{s}$ & $+30^{\circ} 16^{\prime} 59^{\prime \prime} 2$ & Comp. Candidate & Sep $=0.82^{\prime \prime}$ \\
FU Ori & $05^{h} 45^{m} 22.6^{s}$ & $+09^{\circ} 04^{\prime} 12^{\prime \prime} 3$ & Comp. Candidate & Sep $=0.55^{\prime \prime}$ \\
HD 38224 & $05^{h} 44^{m} 30.8^{s}$ & $+08^{\circ} 38^{\prime} 13^{\prime \prime} 9$ & Comp. Candidate & Sep $=1.08^{\prime \prime}$ \\
V 380 Cep & $21^{h} 01^{m} 36.9^{s}$ & $+68^{\circ} 09^{\prime} 47^{\prime \prime} 8$ & Comp. Candidate & Sep $=5.78^{\prime \prime}$ \\
\hline
\end{tabular}




\subsection{Circumstellar Envelopes}

Our PSF-subtracted images show extended emission around the two young stars AB Aur and SU Aur. These extended emissions have been detected previously (see, e.g. Grady 2001) and presumed to originate from light scattering on the circumstellar envelopes. In the case of the ALFA observations of SU Aur this emission can be traced back as far as $14^{\prime \prime}$ from the central star. Our highresolution multi-color near-infrared imaging significantly contributes to the understanding of the structure of these envelopes and are being interpreted as a part of a multi-wavelength observing campaign (Grady et al. 2003).

\subsection{Companion Candidates}

The AO-assisted PSF-subtraction technique is rather efficient in detecting fainter stellar objects close to the bright central stars. We have identified a total of 6 companion candidates (see Table 1.). The current imaging data set is insufficient to determine the nature of these objects but spectroscopic follow-up observations are in preparation.

The most interesting companion candidate is that of FU Ori, which is the prototype of objects that displayed sudden, dramatic increase in luminosity. This brightening is widely believed to be the result of enhanced accretion rates, but the trigger event is yet unknown. A fly-by of a stellar companion could easily serve as trigger, therefore our detection of a companion candidate might be important for understanding the FU Ori-type objects.

\section{NACO Observations of TW Hya}

We used the newly installed NACO/VLT AO-system at Paranal, Chile to image the closest classical T Tauri star TW Hya. In order to study its circumstellar disk as close as possible to the star, the suppression of the stellar light is necessary. We used the method known as polarimetric differential imaging (PDI) (see, e.g. Kuhn, Potter \& Praise 2001). The basic idea of the PDI is to remove the unpolarized light of the star from the images but keep the scattered and therefore polarized light from the disk. In order to realize this concept, we introduced a Wollaston-prism into the light path, that splits the incoming light into an ordinary and an extraordinary beam with orthogonal polarizations. For each integration the difference of these two beams were made to remove the unpolarized light. In order to reduce the effects of the intrumental polarization, redundant data sets with Wollaston angles of $0^{\circ}, 45^{\circ}, 90^{\circ}$ and $135^{\circ}$ were taken. At every Wollaston position a 3-point dithering was applied with steps of about $9^{\prime \prime}$ along the field of view to allow sky subtraction and to reduce the influence of bad pixels. To ensure the high dynamic range, we repeated the complete set of these observations with defined integration times of $0.4 \mathrm{~s}$ and $30 \mathrm{~s}$. The total time spent on the source was $24 \mathrm{~s}$ and $1800 \mathrm{~s}$ in the short and long exposure series, respectively.

The data reduction was carried out using self-developed IDL routines. First, every frame was manually inspected and those showing reflections or electronic ghosts were excluded from the further reduction. Hot pixels were efficiently removed by a 3.5-sigma filtering process. The raw images were sky 

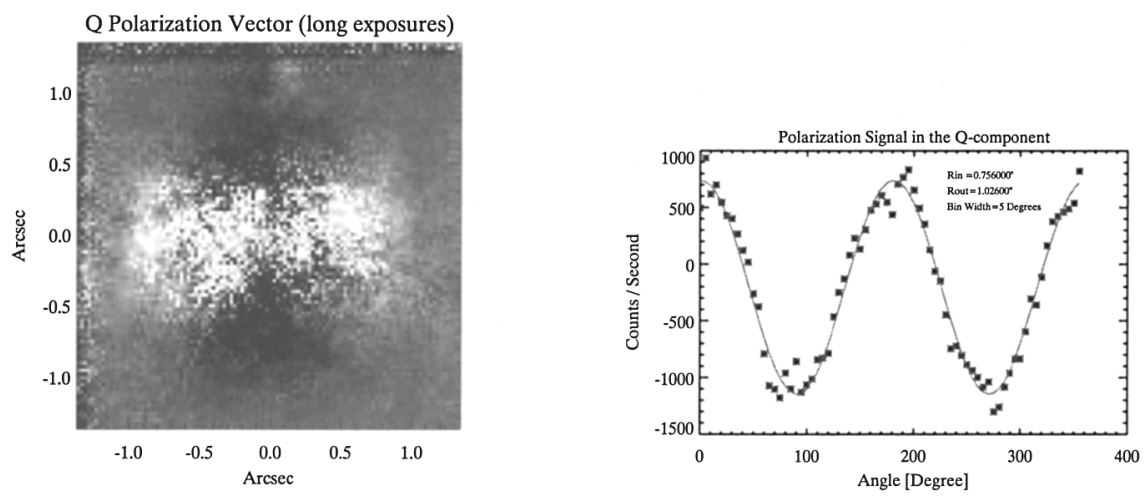

Figure 1. Left panel: The characteristic butterfly pattern of an extended polarized source around TW Hya in the $Q_{\text {Long }}$ image. This pattern can be identified between $0.5^{\prime \prime}$ and $1.4^{\prime \prime}$ on the long exposure image. The image is centered on TW Hya (RA: $11^{\mathrm{h}} 01^{\mathrm{m}} 51.9^{\mathrm{s}}$ Dec: $\left.-34^{\circ} 42^{\prime} 17^{\prime \prime}\right)$. Right panel: Counts in the Q-component of the polarization vector as a function of position angle in an annulus between $0.75^{\prime \prime}$ and $1^{\prime \prime}$ from the long exposure series. The asterisks mark the data points averaged over 5 degree bins, while the curve is the best fitting frequency-fixed cosinus. The strong sinusoidal modulation indicates that the lightscattering dust is distributed nearly axisymmetrically around the light source.

subtracted and flat field corrected. The sky frames were calculated individually for each group of images taken with the same polarization angle. Following these basic corrections, the ordinary and extraordinary peaks of the individual exposures were centered and extracted. To remove all of the non-polarized intensity (mainly from the central star) we subtracted images of ordinary polarization from those of extraordinary polarization. The fine alignment of the individual images before subtraction and co-addition was carried out by a two-level gaussian fitting procedure. To characterize the surface brightness distribution of the disk we measured the radial profile of the polarized intensity, derived from 1-pixel-wide apertures.

\section{NACO Results}

Based on the NACO observations, we obtained Ks-band images of the circumstellar disk around the classical T Tauri star TW Hya, which probe the disk structure closer to the star than any previous observation. Our diffraction limited PDI of TW Hya shows an extended butterfly pattern (see Fig. 1) characteristic of a spatially resolved axisymmetric light scattering source. This pattern is present between $0.5^{\prime \prime}$ and $1.4^{\prime \prime}$ on the long exposure images and between $0.1^{\prime \prime}$ and $0.4^{\prime \prime}$ in the short exposure images. 


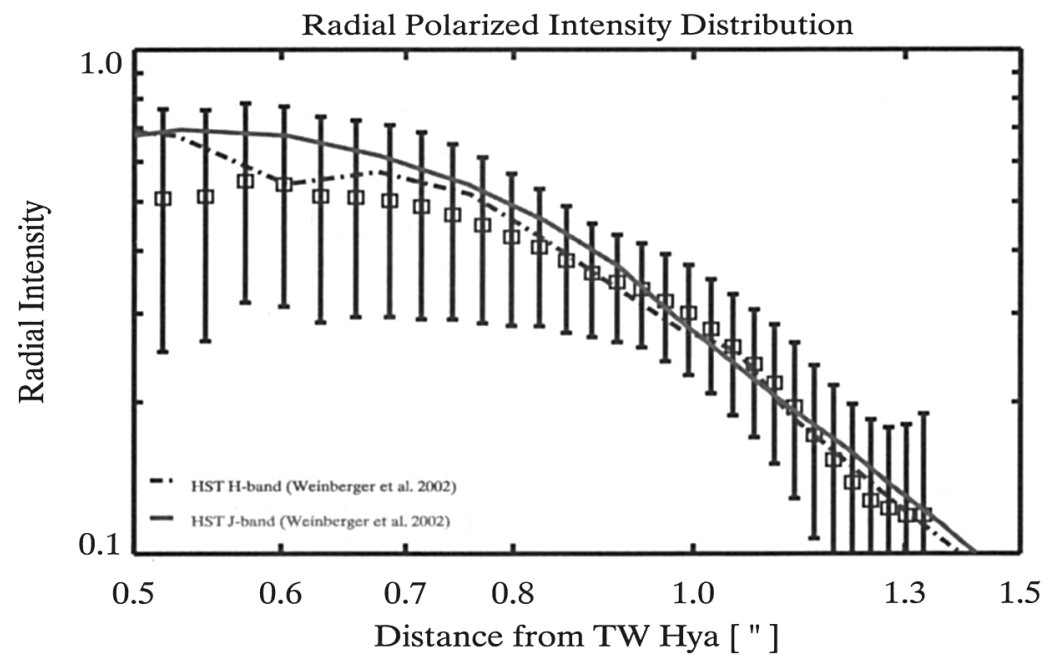

Figure 2. Radial polarized intensity distribution of the TW Hya disk between $0.5^{\prime \prime}$ and $1.4^{\prime \prime}$. The squares mark our Ks-band PDI data, while the solid and dashed curves show the J- and H-band surface brightness distributions from Weinberger et al. (2002). Although the surface brightness is not equivalent to the PI, applying an arbitrary offset shows the similar behaviour of the two curves.

The observations of the regions closer than $0.1^{\prime \prime}$ to the star and between $0.4^{\prime \prime}$ and $0.5^{\prime \prime}$ do not have sufficient signal-to-noise ratio (SNR) for a reliable analysis. Still, the detection of the extended polarized emission as close as $0.1^{\prime \prime} \simeq 6 \mathrm{AU}$ from the star is the first direct evidence that the disk extends so close in and harbors no large inner gap or 'dark zone' of suppressed flaring angle as proposed by Krist et al. (2000). The SNR of the data set, however, is sufficiently high to plot the azimuthaly integrated Ks-band polarized intensity distribution between $0.5^{\prime \prime}$ and $1.4^{\prime \prime}$ (see Fig. 2). This is the longest wavelength at which such a profile has been derived with high-resolution techniques. The profile shows a gradual slope change from the rather steep power-law $P I(r>0.9) \sim r^{-3.1 \pm 0.3}$ to the very gentle $P I(r<0.7) \sim r^{-0.9 \pm 0.2}$. The fact, that a similar behaviour has been found by the HST coronographic observations at shorter wavelengths (Krist et al. 2000, Weinberger et al. 2002) shows that the surface brightness profile is well traced by the polarized intensity profile in the case of the TW Hya disk.

For a more detailed discussion of these observations and results see Apai et al. (2003). We note, that the PDI technique has been recently used to obtain a higher quality data set on numerous targets in the TW Hya Association (see Huélamo \& Brandner 2003).

\section{Conclusions}

Our ALFA adaptive optics observations of young stars led to the first detection of numerous companion candidates, including one around FU Ori. We obtained the first multi-color high-resolution near-infrared images of the nebulosity around SU 
Aur and $\mathrm{AB}$ Aur, which provide important information on these circumstellar envelopes. Furthermore, our polarimetric differential imaging of the classical T Tauri star TW Hya explored its disk closer to the star than any previous observation, up to $0.1^{\prime \prime} \simeq 6 \mathrm{AU}$. This shows, that the combination of the highorder AO system, the $8 \mathrm{~m}$-class telescope and the PDI technique forms a unique observing tool capable of studying disk structure at the scales of our inner Solar System. These results demonstrate the important role of high-contrast and high-resolution imaging in the study of companions, envelopes, disk evolution and structure around young, nearby stars.

\section{References}

Apai, D., Pascucci, I., Brandner, W., Henning, Th., Lenzen, R., Potter, D., Lagrange, A.-M., \& Rousset, G. 2003 A\&A, submitted

Huélamo, N. \& Brandner, W. 2003 in preparation

Krist, J. E., Stapelfeldt, K. R., Ménard, F., Padgett, D. L., \& Burrows, C. J. 2000 ApJ, 538, 793

Kuhn, J. R., Potter, D., \& Praise, B. 2001 ApJ, 553, 189

Grady, C., Stapelfeldt, K., Clampin, M., Padgett, D., Woodgate, B., Henning, Th., Grinin, V., Quirrenbach, A., Stecklum, B., Sitko, M., \& Biggs, J. 2001 AAS, 199, 60.15

Grady, C. et al. 2003, in preparation

Wang, H., Apai, D., Henning, Th., Pascucci, I., \& Leinert, Ch. 2003 ApJ, in preparation

Weinberger, A. J., Becklin, E. E., Schneider, G., Chiang, E. I., Lowrance, P. J., Silverstone, M., Zuckerman, B., Hines, D. C., \& Smith, B. A. 2002 ApJ, 566,409 